\title{
Conditional Effect of the Deletion of eshA on Streptomycin Production in Streptomyces griseus IFO13350
}

\author{
Takeaki Tezuka, Yasuo Ohnishi* and Sueharu Horinouchi ${ }^{\dagger}$ \\ Department of Biotechnology, Graduate School of Agriculture and Life Sciences, The University of Tokyo, \\ Bunkyo-ku, Tokyo 113-8567, Japan \\ (Received Sep. 29, 2010 / Accepted Oct. 22, 2010 / Published Dec. 25, 2010)
}

\begin{abstract}
The $e s h A$ gene was originally found to encode a protein required for the extension of sporogenic hyphae during submerged spore formation in Streptomyces griseus NRRL B-2682. An eshA-disrupted strain of $S$. griseus IFO13189 was reported to be conditionally deficient in streptomycin production and aerial mycelium formation. Our previous transcriptomic analyses indicated that AdpA, a global transcriptional regulator of morphological and physiological differentiation, induced eshA (SGR1270) transcription in $S$. griseus IFO13350. Here, we examined the transcriptional regulation of eshA by AdpA and the involvement of eshA in the morphological and physiological differentiation of S. griseus IFO13350. Transcriptional analysis by $\mathrm{S} 1$ nuclease mapping showed that $e s h A$ was transcribed throughout growth on solid medium. In contrast, no eshA transcription was detected in an adpA deletion mutant. Recombinant His-tagged AdpA bound to a region upstream from the eshA promoter in vitro. However, mutation of the AdpA-binding sequence did not affect the transcription of eshA in vivo, indicating that AdpA indirectly activates $e s h A$ transcription. Streptomycin production by an $e s h A$ deletion mutant grown on TSB plates was lower than that of the wild-type strain. However, the $e s h A$ deletion mutant grew and formed aerial mycelia and spores following the same time course as the wild-type strain on various media.
\end{abstract}

\section{INTRODUCTION}

The morphological development and secondary metabolism of Streptomyces griseus are globally controlled by the chemical signaling molecule A-factor (2-isocapryloyl$3 R$-hydroxymethyl- $\gamma$-butyrolactone) (Horinouchi, 2007; Horinouchi \& Beppu, 2007). AdpA, the central transcriptional regulator in the A-factor regulatory cascade, activates a number of genes required for morphological development and secondary metabolite formation, which together form an AdpA regulon (Ohnishi et al., 2005). We previously determined the complete genomic sequence of S. griseus IFO13350, and, by DNA microarray analysis, the effects of AdpA on global gene expression (Ohnishi et al., 2008). A comparison of the transcriptomes of the wildtype strain and an $a d p A$ deletion $(\triangle a d p A)$ mutant predicted eshA (extension of sporogenic hyphae; SGR1270) to be a possible AdpA-inducible gene (fold-change, 2.3; $p=$ $0.025)$, which prompted us to examine the transcriptional regulation of eshA by AdpA in S. griseus IFO13350.

The $\operatorname{esh} A$ gene was originally found to encode a protein required for the extension of sporogenic hyphae during submerged spore formation in liquid cultures of S. griseus NRRL B-2682 (Kwak et al., 2001). Subsequent analyses revealed that EshA had positive effects on actinorhodin production and aerial mycelium formation in $S$. coelicolor A3(2) 1147 (Kawamoto et al., 2001; Saito et al., 2006). An eshA disrupted strain accumulated lower levels of ppGpp than the corresponding wild-type strain (Saito et al., 2006). EshA contains a cyclic nucleotide-binding domain that is indispensable for its activation of actinorhodin production (Saito et al., 2006). An eshA mutant strain of S. griseus IFO13189 was found to be conditionally deficient in streptomycin production and aerial mycelium formation, and displayed a lower rate of DNA synthesis than the wild-type strain (Saito et al., 2003). EshA localizes to the cytoplasm, where it forms large multimeric cube-like structures (Saito et al., 2003). Though many studies have sought to characterize EshA genetically and biochemically, its functional roles across Streptomyces species have not yet been fully elucidated. We therefore examined the effect of deletion of eshA on morphogenesis and secondary metabolism in S. griseus IFO13350.

\section{MATERIALS AND METHODS}

General recombinant DNA studies

The restriction enzymes, T4 DNA ligase, and other DNA-modifying enzymes used in this study, were purchased from Takara Biochemicals. $\left[\alpha-{ }^{32} \mathrm{P}\right] \mathrm{dCTP} \quad(110$

\footnotetext{
${ }^{*}$ Corresponding author. Mailing address: Department of Biotechnology, Graduate School of Agriculture and Life Sciences, The University of Tokyo, Bunkyo-ku, Tokyo 113-8657, Japan. Tel: 813 5841-5123; Fax: 813 5841-8021; E-mail: ayasuo@mail.ecc. u-tokyo.ac.jp

${ }^{\dagger}$ Deceased on 12th July 2009
} 
$\mathrm{TBq} / \mathrm{mmol}$ ) (for DNA labeling with the BcaBest DNA labeling system [Takara Biochemicals]) and $\left[\gamma_{-}{ }^{32} \mathrm{P}\right] \mathrm{ATP}$ $\left(220 \mathrm{TBq} / \mathrm{mmol}\right.$ ) (for $5^{\prime}$-end labeling with $\mathrm{T} 4$ polynucleotide kinase) were purchased from PerkinElmer. DNA was manipulated in Streptomyces (Kieser et al., 2000) and Escherichia coli (Ausubel et al., 1987; Maniatis et al., 2001) using previously described protocols. PCR products were confirmed to be error-free by DNA sequencing following cloning into pUC19 vector. Nucleotide sequences were determined by the dideoxy chain-termination method using a Thermo Sequenase fluorescence-labeled primer cycle sequencing kit (GE Healthcare).

\section{Bacterial strains, media and plasmids}

S. griseus IFO13350 was obtained from the Institute of Fermentation (Osaka, Japan). The $S$. griseus mutant $\triangle a d p A$ has been described previously (Ohnishi et al., 1999). The Streptomyces strains were grown in YMPD medium. YMPD agar contained 2.2\% agar (Tezuka et al., 2009). R2YE medium (Kieser et al., 2000) was used for protoplast regeneration. Bennett, SMM (Hirano et al., 2008) and TSB (Saito et al., 2003) media were also used in this study. Neomycin $(20 \mu \mathrm{g} / \mathrm{ml})$ and thiostrepton $(20 \mu \mathrm{g} / \mathrm{ml})$ were added when necessary. E. coli JM109 and the vector pUC19 (for use in DNA manipulation) were purchased from Takara Biochemicals. E. coli JM110 cells carrying dam and $d \mathrm{~cm}$ mutations were used to prepare nonmethylated Streptomyces DNA for gene disruption. Histagged AdpA was purified from E. coli BL21(DE3) harboring pET-adpA as described previously (Yamazaki et al., 2000). The media and growth conditions for E. coli culture were as described by Maniatis et al. (2001). Ampicillin $(50 \mu \mathrm{g} / \mathrm{ml})$ and kanamycin $(50 \mu \mathrm{g} / \mathrm{ml})$ were used when necessary.

\section{Plasmid construction}

For cloning of the DNA fragment containing the esh $A$ coding sequence and its upstream region, we first amplified this region by PCR using the $S$. griseus chromosome as template and the primers GF (5'-CCGGAATTCTCTACCCTGTTCCGCGCGTCGAC-3' [EcoRI site underlined]) and ACR (5'-GCCAAGCTTTGGACTCCTTGATGAGGTGTG-3' [HindIII site underlined]). The amplified fragment was digested with EcoRI and HindIII and cloned between the EcoRI and HindIII sites in pUC19, generating pUC-eshAwt. The EcoRI-HindIII fragment was then excised from pUC-eshAwt and cloned into the same sites in the integration vector pTYM19 (Onaka et al., 2003), yielding pTYM-eshAwt.

A mutation was introduced into AdpA-binding site 2, located upstream from the eshA promoter. The sequence CTGAAA in site 2 was replaced with the BamHI cleavage sequence GGATCC by PCR using pUC-eshAwt as the template (see Fig. 2A). A 140-bp DNA fragment containing the region upstream of the AdpA-binding site 2 was amplified with the primers GF and M2-R (5'-CGGGATCC-
CCGTGATCGAGGAGGGCTTT-3' [BamHI site underlined]), and then digested with EcoRI and BamHI. Separately, a 1,733-bp DNA fragment containing the region downstream of the AdpA-binding site 2 was amplified with the primers M2-F (5'-CGGGATCCCGCGTCGGCCCGGGGGCAAA-3' [BamHI site underlined]) and ACR and then digested with BamHI and HindIII. The 133-bp EcoRI-BamHI fragment and the 1,726-bp BamHI$H i n d I I I$ fragment were inserted between the EcoRI and HindIII sites in pUC19 by three-fragment ligation, generating pUC-eshAm2. Finally, the 1,855-bp EcoRI-HindIII fragment was excised from pUC-eshAm2 and cloned into the same sites in pTYM19, generating pTYM-eshAm2.

\section{RNA isolation}

Total RNA was isolated from cells grown at $28^{\circ} \mathrm{C}$ on cellophane on the surface of YMPD agar medium using ISOGEN (Nippon Gene) as described previously (Tezuka et al., 2009).

\section{S1 nuclease mapping}

S1 nuclease mapping was performed as described by Bibb et al. (1986) and Kelemen et al. (1998). Hybridization probes were prepared by PCR with ${ }^{32} \mathrm{P}$-labeled/ unlabeled primer pairs. $h r d B$, which encodes the principal sigma factor of RNA polymerase, was used to determine the amount and purity of RNA used, as described previously (Ohnishi et al., 1999). The PCR primers used for the eshA probe were AF (5'-TACGCGGGTGCTCTACCCTGTTC- $3^{\prime}$ ) and AR (5'-GAGGCATGCATAGCGGACATGAC-3'). The PCR primers used for the eshB probe were BF (5'-CACGTCGGTGAATCTCGTCAGCC$\left.3^{\prime}\right)$ and BR (5'-TCACCAACGGACATCTGACGTCC-3'). Primers AR and BR were $5^{\prime}$-labeled with $\left[\gamma_{-}{ }^{32} \mathrm{P}\right] \mathrm{ATP}$ using T4 polynucleotide kinase prior to their use in PCR. Marker 10 (pBR322/MspI digest, Nippon Gene) was 5'-labeled with $\left[\gamma_{-}{ }^{32} \mathrm{P}\right] \mathrm{ATP}$ and used as a DNA standard marker.

\section{Gel mobility shift assay}

The purification of His-tagged AdpA from E. coli BL21(DE3) cells and gel mobility shift assays were performed as described previously (Yamazaki et al., 2000). The DNA fragments used as probes were amplified by PCR and ${ }^{32} \mathrm{P}$-labeled with $\mathrm{T} 4$ polynucleotide kinase. All probes were designed to the region upstream of the $e s h A$ coding sequence.

\section{Mutation of the AdpA-binding sequences by PCR}

Mutations were introduced into the AdpA-binding sites by PCR. A $0.2-\mathrm{kb}$ fragment (positions -232 to +10 relative to the transcription start site in eshA) was amplified by PCR using the primers GF and GR (5'-GCCAAGCTTCCAGATCCAGGGGAACACTAAGG-3' [HindIII site underlined]) using the $S$. griseus chromosome as template. The amplified fragment was digested with EcoRI and HindIII and cloned between the EcoRI and HindIII sites in pUC19, generating pUC-eshAp. The 252-bp EcoRI-HindIII fragment was 
excised from pUC-eshAp and used in gel mobility shift assays as an intact probe (probe $\mathrm{W}$ ). The sequence CCGGAT in AdpA-binding site 1 was replaced with the $K p n I$ cleavage sequence GGTACC by PCR using pUC-eshAp as the template (see Fig. 2A). A 65-bp DNA fragment containing the region upstream of the AdpA-binding site 1 was amplified with primers GF and M1-R (5'-GGGGTACCCCGGTCGGAGCGGGTGGGCG-3' ${ }^{\prime}$ KpnI site underlined]) and digested with EcoRI and KpnI. A 205-bp DNA fragment containing the region downstream of the AdpAbinding site 1 was amplified with primers M1-F (5'-GGGGTACCTTCGCCGGGCATTCGCCGCT-3' [KpnI site underlined]) and GR and then digested with $K p n \mathrm{I}$ and HindIII. The 58-bp EcoRI-KpnI fragment and 198-bp KpnIHindIII fragment were inserted between the EcoRI and HindIII sites in pUC19 by three-fragment ligation, generating pUC-eshAml. The 252-bp EcoRI-HindIII fragment was then excised from pUC-eshAml and used in gel mobility shift assays as the mutated probe M1. The six nucleotides in AdpA-binding sites 2 and 3 were similarly replaced with Bam $\mathrm{HI}$ and PstI recognition sequences by PCR and used as the mutated probes M2 and M3, respectively (see Fig. 2A).

\section{Gene disruption}

To generate disruptants, most of the $e s h A$ or $e s h B$ coding sequence was deleted from the chromosome of $S$. griseus. Sequences from regions upstream and downstream of the coding region (each approximately $2 \mathrm{~kb}$ in length) were assembled in pUC19, together with the neomycin resistance gene aphII. The primers used for the eshA upstream region were AUF (5'-GCCAAGCTTTCCCCGTCATGGTCGACAACGAC-3' [HindIII site underlined]) and AUR (5'CGCGGATCCAACAGTCATCGGGCAGGCTCTCC-3' [BamHI site underlined]), while those for the eshA downstream region were ADF (5'-CGCGGATCCCAGATCGCCAACTGGCCCAGGTAG-3' [BamHI site underlined]) and ADR (5'-CCGGAATTCGGTCCACGTACATGCTCGACTCG-3' [EcoRI site underlined]). The primers used for the $e s h B$ upstream region were BUF (5'-TTTCCTGCAGGCCGAACACCTCGTAGTGGACGTC-3' [Sse8387I site underlined]) and BUR (5'-GCTCTAGAGCGAACCTCTTCACCAACGGA-3' [XbaI site underlined]), while those for the $e s h B$ downstream region were BDF (5'-CGTCTAGAGTGCTGGAGAACGTCGAGGTCGG-3' [XbaI site underlined]) and BDR (5'-CCCTTGTTGTCCCGGTACCAGCC-3'). The aphII gene cassette was inserted into the $H i n$ dIII and Sse8387I sites on the eshA and $e s h B$ disruption plasmids, respectively. The plasmids were introduced by protoplast transformation into S. griseus, and neomycinresistant transformants resulting from a single crossover of the plasmid into the chromosome were isolated. Neomycinsensitive colonies, derived from a second crossover in one of the transformants, were identified as candidate eshA/ eshB disruption strains. The correct replacements were confirmed by Southern hybridization using each gene and aphII as ${ }^{32} \mathrm{P}$-labeled probes.

\section{Streptomycin assay}

The amount of streptomycin produced was measured in a bioassay in which Bacillus subtilis was used as the indicator (Horinouchi et al., 1984).

\section{RESULTS}

\section{Expression profiles of $e s h A$ and $e s h B$ in the wild-type and $a d p A$-disrupted strains}

A comparison of the transcriptomes in the wild-type and $\triangle a d p A$ strains grown in YMPD liquid medium identified eshA (SGR1270) as a putative AdpA-inducible gene (foldchange, $2.3 ; p=0.025)$. S. griseus IFO13350 has an eshA homolog, eshB (SGR2264). The amino acid sequence of EshB shows $64 \%$ identity with that of EshA. Because the transcription of $e s h B$ was suggested in the same analysis to be induced by AdpA (fold-change, 2.0; $p=0.026$ ), we examined the transcription of $e s h A$ and $e s h B$ in the wildtype and $\triangle a d p A$ strains by low-resolution $\mathrm{S} 1$ nuclease mapping. For this analysis, total RNA was isolated from S. griseus cells grown on YMPD agar at $28^{\circ} \mathrm{C}$ for 24,48 and $72 \mathrm{~h}$. On YMPD solid medium, the wild-type strain grew as substrate mycelia at $24 \mathrm{~h}$, as a mixture of substrate and aerial mycelia at $48 \mathrm{~h}$, and as a mixture of substrate mycelia and aerial hyphae with spores at $72 \mathrm{~h}$. The $\triangle a d p A$ mutant grew as substrate mycelia throughout the time course. In the wild-type strain, eshA transcription was detected throughout the time course; its expression appeared to decrease at $48 \mathrm{~h}$, before increasing again at $72 \mathrm{~h}$ (Fig. 1). An additional transcript with a different 5 -end was also detected at 48 and $72 \mathrm{~h}$. The approximate transcription start site in $e s h A$ (predicted from the larger band) agreed with the precise start point reported previously (Kwak et al., 2001). The transcription of eshB was detected at 48 and $72 \mathrm{~h}$, but not at $24 \mathrm{~h}$. Importantly, in the $\triangle a d p A$ mutant, the transcription of eshA and eshB was completely lost, suggesting the possible involvement of AdpA in the transcriptional activation of these two genes.

\section{Effect of AdpA on eshA expression}

We examined AdpA binding to the upstream region of eshA in a gel mobility shift assay using recombinant Histagged AdpA purified from E. coli. The ${ }^{32} \mathrm{P}$-labeled probe tested in this assay (positions -232 to +10 relative to the transcription start site in $e s h A$ ), designated $\mathrm{W}$, yielded a shifted band (Fig. 2A and B). When we searched region upstream from the $e s h A$ promoter for the AdpA-binding consensus sequence ( $5^{\prime}$-TGGCSNGWWY-3', where $\mathrm{S}=\mathrm{G}$ or $\mathrm{C} ; \mathrm{W}=\mathrm{A}$ or $\mathrm{G} ; \mathrm{Y}=\mathrm{T}$ or $\mathrm{C} ; \mathrm{N}=$ any nucleotide) (Yamazaki et al., 2004), allowing for up to three mismatches, we identified three possible sequences, which were denoted sites 1-3 (Fig. 2A). We introduced mutations into these three possible AdpA-binding sequences to replace the original six nucleotides with $K p n \mathrm{I}, B a m \mathrm{HI}$ and PstI recognition sequences, yielding the mutated probes M1, M2 and M3, respectively (Fig. 2A). The effect of these 


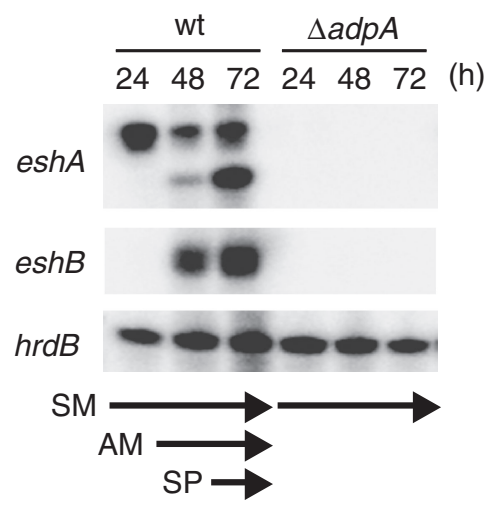

Fig. 1. Time course of $e s h A$ and $e s h B$ transcription as determined by low-resolution S1 nuclease mapping. RNA was prepared from wild-type and $\triangle a d p A$ cells grown at $28^{\circ} \mathrm{C}$ on cellophane on the surface of YMPD agar for the indicated time periods. $h r d B$ was used as an internal control. SM, substrate mycelium; AM, aerial mycelium; SP, spore; wt, wild-type. The $\triangle a d p A$ mutant grew only as substrate mycelia throughout the growth period. mutations on AdpA binding was examined by a gel mobility shift assay. The M2 probe containing the mutation in site 2 produced no signal retardation, whereas the other two mutated probes yielded shifted bands that were similar to those produced by the intact probe (probe W). This result indicated that site 2 (positions -103 to -112 relative to the transcription start site in $e s h A$ ) is important for the binding of AdpA to this region.

To evaluate the importance of the binding of AdpA to the upstream region of $e s h A$ for the transcription of $e s h A$ in vivo, eshA with a mutation in site 2 was integrated into the chromosome of an eshA deletion mutant using the integration vector pTYM19 (the eshA deletion mutant is described below). The eshA gene with an intact site 2 was also integrated into the chromosome as a control. RNA was extracted from $\triangle e s h A$ mutants carrying the wild-type and site 2-mutated forms of $e s h A$, which were grown for 24, 48 and $72 \mathrm{~h}$ on solid YMPD medium, and were then analyzed for eshA expression by $\mathrm{S} 1$ nuclease mapping. Contrary to our expectation, the levels of $e s h A$ transcription were similar in the two strains (Fig. 2C). This result indicated that the binding of AdpA to the upstream region of $e s h A$ did not have a significant effect on eshA transcription. There-
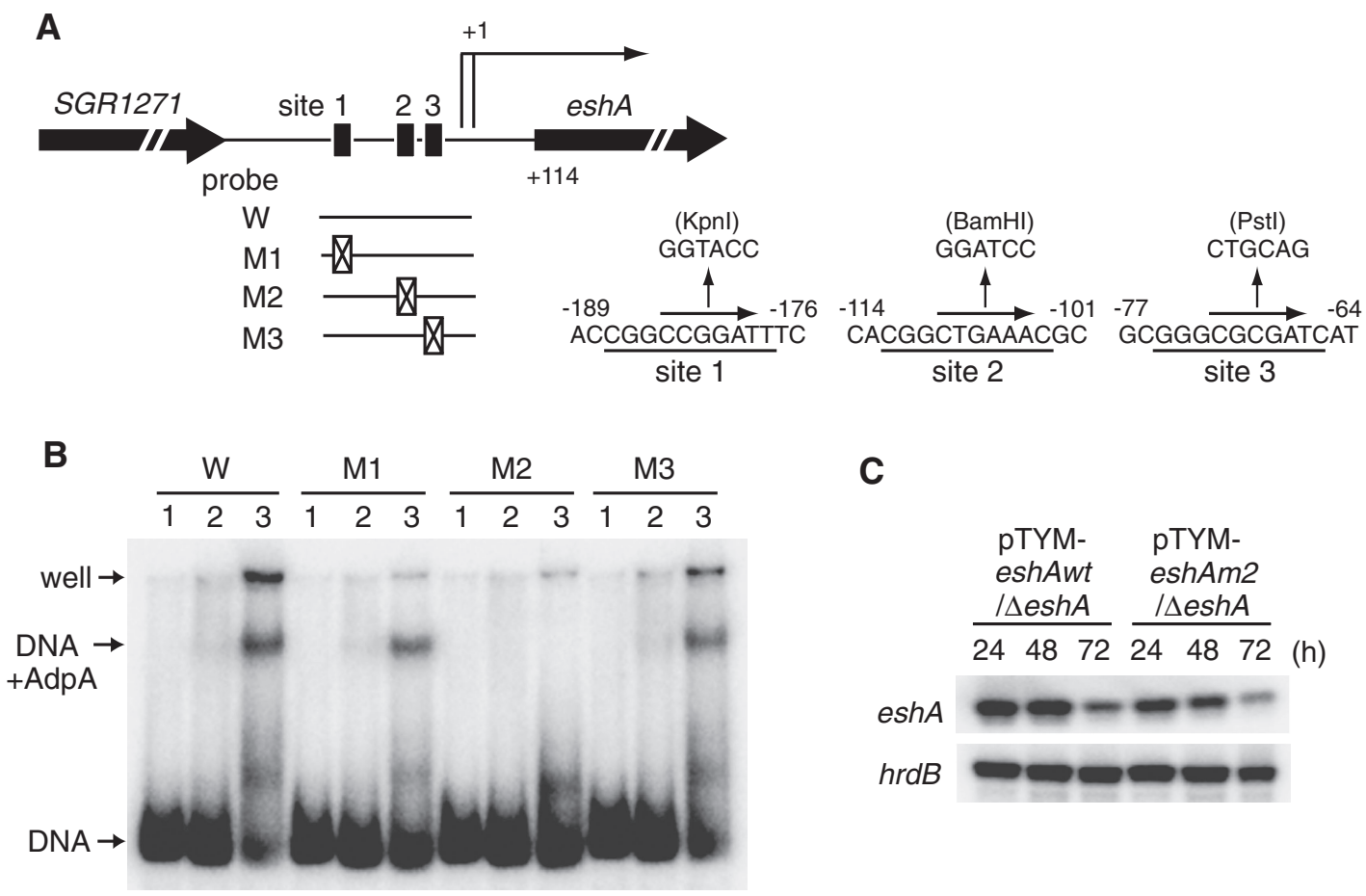

C

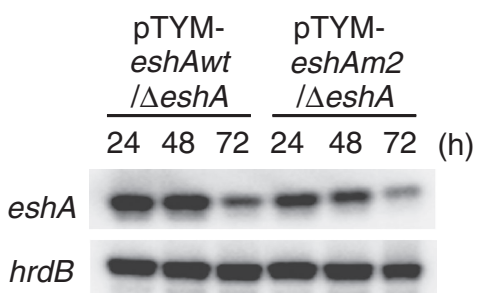

Fig. 2. AdpA binding to the region upstream from the eshA promoter. A) Schematic representation of the possible AdpA-binding sites in the region upstream of $e s h A$, the probes used in B), and the mutations introduced into each site. Mutations were introduced into the putative AdpA-binding sequences (sites 1-3) by PCR to replace the original six nucleotides with KpnI, BamHI and PstI recognition sites, respectively. The putative AdpA-binding sequence in each site is underlined. B) A gel mobility shift assay was performed using purified AdpA and ${ }^{32} \mathrm{P}$-labeled DNA probes. Probe W, containing the intact sequence of the upstream region of the eshA gene (from -232 to +10 ), was used. The following amounts of AdpA were used: $0.2 \mu \mathrm{g}$ (lane 2) and $0.4 \mu \mathrm{g}$ (lane 3). Lane 1: negative control (no AdpA). Three mutated probes (M1, M2 and M3) were also used. C) Time course of eshA transcription as determined by low-resolution S1 nuclease mapping with RNA prepared from $\Delta e s h A$ mutant cells harboring pTYM-eshAwt or pTYM-eshAm2 and grown at $28^{\circ} \mathrm{C}$ on cellophane on the surface of YMPD agar for the indicated time periods. $h r d B$ transcription was assessed as an internal control. 
fore, we conclude that AdpA does not directly activate eshA. The molecular mechanism underlying the absence of $e s h A$ transcription in the $\triangle a d p A$ mutant remains to be elucidated.

\section{Functional analysis of $e s h A$ and $e s h B$}

To investigate the in vivo function of eshA, most of the $e s h A$ coding sequence was deleted without inserting a marker gene to avoid possible polar effects on the expression of the flanking open reading frames. Correct disruption of the gene was confirmed by Southern hybridization using appropriate probes (data not shown). First, the $e s h A$ deletion mutant $(\triangle e s h A)$ was incubated at $28^{\circ} \mathrm{C}$ on various media (YMPD, R2YE, SMM, Bennett and TSB agar). No difference in morphological differentiation was observed between the wild-type and $\Delta e s h A$ strains. Next, we examined the production of streptomycin, a representative secondary metabolite produced by $S$. griseus, on glucose-depleted Bennett agar and TSB agar, using $B$. subtilis as an indicator. The growth-inhibition zones surrounding the five- and six-day-old $\Delta e s h A$ colonies on TSB plates were smaller than those of the wild-type strain (Fig. 3). We repeated this assay three times and calculated the amount of streptomycin produced. The wild-type strain produced $3.0 \pm 0$ and $8.7 \pm 0.1 \mu \mathrm{g}$ of streptomycin per colony on days 5 and 6 , respectively, whereas the $\Delta e s h A$ mutant produced only $1.1 \pm 0$ and $1.7 \pm 0.1 \mu \mathrm{g}$ of streptomycin per colony on days 5 and 6 , respectively. The reduced streptomycin production by the $\triangle e s h A$ mutant was almost completely compensated by the integration of eshA with its own promoter into the $S$. griseus $\triangle$ eshA chromosome. Mutant $\triangle e s h A$ harboring pTYM19-eshAwt produced $2.4 \pm 0$ and $6.2 \pm 0.1 \mu \mathrm{g}$ of streptomycin per colony on days 5 and 6 , respectively $(n=3)$. In contrast, no differences in streptomycin production were observed between the wild-type and $\Delta e s h A$ strains on glucose-depleted Bennett agar. These data showed that EshA exerts a positive, but conditional, effect on streptomycin production.

In addition to the $\triangle e s h A$ mutant, we generated an $e s h B$ deletion $(\Delta e s h B)$ mutant and a double $(\Delta e s h A \Delta e s h B)$ mutant. However, no phenotypic differences were observed

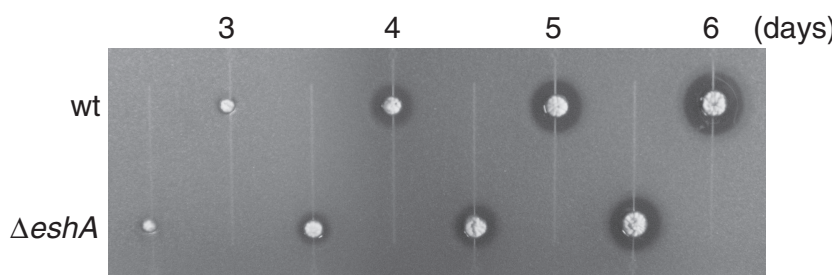

Fig. 3. Streptomycin production by the $\Delta e s h A$ mutant. Wildtype and $\triangle e s h A$ cells were grown at $28^{\circ} \mathrm{C}$ on TSB agar for the indicated number of days, and were then overlaid with soft agar containing B. subtilis spores. Following incubation of the plates overnight at $28^{\circ} \mathrm{C}$, streptomycin production was detected as the zone of growth inhibition of the indicator surrounding each colony. wt, wild-type. between the wild-type and $\triangle e s h B$ strains, or between the $\triangle e s h A$ and $\triangle e s h A \Delta e s h B$ mutants. These results indicated that EshB exerts no apparent effect on morphological differentiation or secondary metabolite formation, at least under the culture conditions used in this study.

\section{DISCUSSION}

In this study, we examined the transcriptional regulation of $e s h A$ by AdpA and the involvement of eshA in the morphological and physiological development of $S$. griseus IFO13350. Contrary to our expectation, the binding of AdpA to the upstream region of $e s h A$ was not responsible for the transcriptional activation of eshA. It is noteworthy that the affinity of AdpA for the upstream region of $e s h A$ was low compared with the affinities of AdpA to other functional AdpA-binding sites. However, the affinity of AdpA to an AdpA-binding site does not necessarily represent the importance of the AdpA-binding site in transcriptional regulation of its neighboring gene. For example, although the affinity of AdpA to the upstream region of $a d s A$ is low, AdpA-binding to this site is essential for the transcriptional activation of adsA (Yamazaki et al., 2000). Therefore, it was necessary to examine the importance of the binding of AdpA to the upstream region of eshA by mutational analysis. Recently, we also found that the binding of AdpA to a region upstream from the $S G R 2418$ (bldK) promoter was not responsible for the activation of that promoter (Akanuma et al., manuscript submitted). These results suggested that the $S$. griseus chromosome contains many AdpA-binding sites with little or no role in the transcriptional regulation of neighboring genes. The suspected involvement of AdpA binding in the activation of a putative target promoter should therefore be confirmed by mutational analyses.

Saito et al. (2003) found that the disruption of eshA abolished aerial mycelium formation in S. griseus IFO13189 grown on TSB agar. However, this phenotype was not observed in S. griseus IFO13350. Although it has been reported that EshA is only produced during submerged spore formation in S. griseus NRRL B-2682 (Kwak et al., 2001) and during late exponential growth and stationary phase in S. griseus IFO13189 (Kawamoto et al., 2001), we detected eshA transcription during vegetative growth in S. griseus IFO13350 on YMPD agar (Fig. 1). In S. griseus IFO13350 grown in liquid YMPD medium, eshA transcription was also detected at 24 (mid-exponential growth phase) and $48 \mathrm{~h}$ (transition phase) (data not shown). These conflicting findings may be ascribed to inter-strain differences. The fact that $S$. griseus IFO13189 has a greater ability to form aerial mycelium and spore than $S$. griseus IFO13350 may be the cause of the phenotypic differences observed between these two strains.

Streptomycin production was markedly reduced in the $\triangle e s h A$ mutant grown on TSB agar. We assume that this is an indirect effect of the $e s h A$ deletion, because the difference in 
streptomycin production between the wild-type and $\Delta e s h A$ strains was observed only on TSB agar and not replicated when the two strains were grown on glucose-depleted Bennett agar. Although the TSB agar used in this study contained $10.3 \%$ sucrose and the glucose-depleted Bennett agar contained no sucrose, high osmotic pressure seemed not to be a critical factor in the phenotypic difference between the wild-type and $\triangle e s h A$ strains on TSB agar; both strains produced almost the same amount of streptomycin on glucose-depleted Bennett agar containing $10.3 \%$ sucrose (data not shown). Glucose-depleted Bennett agar is used routinely in streptomycin production assays in our laboratory. Saito et al. reported that in $S$. coelicolor A3(2), an eshA disruptant accumulated lower levels of ppGpp than the parent strain (Saito et al., 2006). The reduced streptomycin production by the $\triangle e s h A$ strain on TSB agar may also be ascribed to lower levels of ppGpp in $S$. griseus.

Close homologs of eshA are widely distributed among Streptomyces species. A BLAST search using the $S$. griseus EshA primary sequence to query 24 Streptomyces genomic sequences registered in the NCBI genome database revealed that all of them contained at least one close homolog of $e s h A$. The broad distribution of the eshA gene among Streptomyces species suggests that eshA has an important function, probably in the morphological and physiological differentiation of members of this genus. However, the loss of eshA function appears not to cause any apparent phenotypic changes under normal growth conditions. Further biochemical characterization of EshA may provide clues as to the culture conditions under which the effect of the deletion of eshA results in dramatic phenotypic changes.

\section{ACKNOWLEDGMENTS}

T. Tezuka was supported by the Japan Society for the Promotion of Science.

\section{REFERENCES}

Ausubel, F.M., Brent, R., Kingstone, R.E., Moore, D.O., Seidman, J.S., Smith, J.A. and Struhl, K. (1987). Current protocols in molecular biology. John Wiley \& Sons, Inc., New York.

Bibb, M.J., Janssen, G.R. and Ward, J.M. (1986). Cloning and analysis of the promoter region of the erythromycin resistance gene (ermE) of Streptomyces erythraeus. Gene 41, 357-368.

Hirano, S., Tanaka, K., Ohnishi, Y. and Horinouchi, S. (2008). Conditionally positive effect of the TetR-family transcriptional regulator AtrA on streptomycin production by Streptomyces griseus. Microbiology 154, 905-914.

Horinouchi, S. (2007). Mining and polishing of the treasure trove in the bacterial genus Streptomyces. Biosci. Biotechnol. Biochem. 71, 283-299.

Horinouchi, S. and Beppu, T. (2007). Hormonal control by Afactor of morphological development and secondary metabolism in Streptomyces. Proc. Jpn. Acad. Ser. B. 83, 277-295.

Horinouchi, S., Kumada, Y. and Beppu, T. (1984). Unstable genetic determinant of A-factor biosynthesis in streptomycinproducing organisms: cloning and characterization. J. Bacteriol. 158, 481-487.

Kawamoto, S., Watanabe, M., Saito, N., Hesketh, A., Vachalova, K., Matsubara, K. and Ochi, K. (2001). Molecular and functional analyses of the gene (eshA) encoding the 52kilodalton protein of Streptomyces coelicolor A3(2) required for antibiotic production. J. Bacteriol. 183, 6009-6016.

Kelemen, G.H., Brian, P., Flärdh, K., Chamberlin, L., Chater, K.F. and Buttner, M.J. (1998). Developmental regulation of transcription of whiE, a locus specifying the polyketide spore pigment in Streptomyces coelicolor A3(2). J. Bacteriol. 180, 2515-2521.

Kieser, T., Bibb, M.J., Buttner, M.J., Chater, K.F. and Hopwood, D.A. (2000). Practical Streptomyces Genetics. Norwich, UK: The John Innes Foundation.

Kwak, J., McCue, L.A., Trczianka, K. and Kendrick, K.E. (2001). Identification and characterization of a developmentally regulated protein, EshA, required for sporogenic hyphal branches in Streptomyces griseus. J. Bacteriol. 183, 3004-3015.

Maniatis, T., Fritsch, E.F. and Sambrook, J. (2001). Molecular Cloning: A Laboratory Manual. Cold Spring Harbor, NY: Cold Spring Harbor Laboratory Press.

Ohnishi, Y., Ishikawa, J., Hara, H., Suzuki, H., Ikenoya, M., Ikeda, H., Yamashita, A., Hattori, M. and Horinouchi, S. (2008). Genome sequence of the streptomycin-producing microorganism Streptomyces griseus IFO 13350. J. Bacteriol. 190, 4050-4060.

Ohnishi, Y., Kameyama, S., Onaka, H. and Horinouchi S. (1999). The A-factor regulatory cascade leading to streptomycin biosynthesis in Streptomyces griseus: identification of a target gene of the A-factor receptor. Mol. Microbiol. 34, 102-111.

Ohnishi, Y., Yamazaki, H., Kato, J.Y., Tomono, A. and Horinouchi, S. (2005). AdpA, a central transcriptional regulator in the A-factor regulatory cascade that leads to morphological development and secondary metabolism in Streptomyces griseus. Biosci. Biotechnol. Biochem. 69, 431-439.

Onaka, H., Taniguchi, S., Ikeda, H., Igarashi, Y. and Furumai, T. (2003). pTOYAMAcos, pTYM18, and pTYM19, actinomycete-Escherichia coli integration vectors for heterologous gene expression. J. Antibiot. 56, 950-956.

Saito, N., Matsubara, K., Watanabe, M., Kato, F. and Ochi, K. (2003). Genetic and biochemical characterization of EshA, a protein that forms large multimers and affects developmental processes in Streptomyces griseus. J. Biol. Chem. 278, 5902-5911.

Saito, N., Xu, J., Hosaka, T., Okamoto, S., Aoki, H., Bibb, M.J. and Ochi, K. (2006). EshA accentuates ppGpp accumulation and is conditionally required for antibiotic production in Streptomyces coelicolor A3(2). J. Bacteriol. 188, 4952-4961.

Tezuka, T., Hara, H., Ohnishi, Y. and Horinouchi, S. (2009). Identification and gene disruption of small noncoding RNAs in Streptomyces griseus. J. Bacteriol. 191, 4896-4904.

Yamazaki, H., Ohnishi, Y. and Horinouchi, S. (2000). An A-factor-dependent extracytoplasmic function sigma factor $\left(\sigma^{\text {AdsA }}\right)$ that is essential for morphological development in Streptomyces griseus. J. Bacteriol. 182, 4596-4605.

Yamazaki, H., Tomono, A., Ohnishi, Y. and Horinouchi, S. (2004). DNA-binding specificity of AdpA, a transcriptional activator in the A-factor regulatory cascade in Streptomyces griseus. Mol. Microbiol. 53, 555-572. 